\title{
The Patient Speaks: Importance of Patient Perspectives in Clinical Decision-Making
}

\author{
Sophia K. McKinley, MD, EDM' ${ }^{1}$, and Genevieve M. Boland, MD, $\mathrm{PhD}^{2}$ \\ ${ }^{1}$ Department of Surgery, Massachusetts General Hospital, Boston; ${ }^{2}$ Department of Surgery, Harvard Medical School, \\ Massachusetts General Hospital, Boston
}

The article by Banting et al. is a small but elegant study examining patient perspectives on the sentinel node biopsy procedure in melanoma in patients with a negative sentinel lymph node. ${ }^{1}$ Of relevance, this represents the vast majority of melanoma patients in whom the sentinel lymph node biopsy procedure is done and in whom the prognostic information of the sentinel node biopsy procedures places them in a low-risk category. For patients with a positive sentinel lymph node, the value of the intervention is clear: early detection, closer surveillance, and potentially additional adjuvant therapy. However, for the large proportion of patients with a negative sentinel lymph node, the "value" of the procedure is not as clear. This study assessed more than 100 patients for physical and functional well-being via measurement of lymphedema, wound complications, and quality of life (QOL). There was heterogeneity to the time points assessed, and this was not a longitudinal study; however, the information obtained was quite informative.

Of the 102 patients evaluated, $19 \%$ were seen within 3 months, $13 \%$ between 4 and 6 months, $21 \%$ between 7 and 12 months, $24 \%$ between 1 and 2 years, and $25 \%$ more than 2 years after their procedure. Only $2 \%$ of all patients had lymphedema, and $25 \%$ had mild (Clavian Grade I/II) wound complications. Overall, physical well-being scores were lower in the first 3 months postsurgery but were otherwise equal across the other time points. Females overall also had a lower QOL score compared with males.

(C) Society of Surgical Oncology 2019

First Received: 11 June 2019;

Published Online: 27 June 2019

G. M. Boland, $\mathrm{MD}, \mathrm{PhD}$

e-mail: gmboland@partners.org
The functional well-being scores also were lower in the first 3 months and with a trend towards ongoing improvement aside from patients with ulcerated tumors. Most importantly, $89 \%$ of patients agreed or strongly agreed that "If they were diagnosed with the same melanoma again, they would choose to undergo sentinel lymph node biopsy."

While the study is limited by a lack of comparator group of non-SLNB patients and does not offer longitudinal analysis within the same patients, it addresses a topic largely avoided until now: what is the value of the sentinel node biopsy procedure in patients who do not benefit from the early detection and aggressive management of melanoma, but instead fall into the low-risk category? Are there psychological or functional benefits to the information associated with negative sentinel node biopsy status? While many of us counsel our patients that the findings of a negative sentinel lymph node may be associated with "peace of mind," very little empiric data have been available to support this statement. This study offers us a window into the patient perspective and confirms the value of the sentinel lymph node procedure, both for those in whom microscopic disease is found, and for those in whom, thankfully, it is not.

The importance of the patient's perspective has been increasing in visibility since the Institute of Medicine issued a 2001 report "Crossing the Quality Chasm," which called for a new phase of the health care in which patient preferences and values critically inform medical decisionmaking. ${ }^{2}$ The concept of patient-centeredness is particularly relevant in cancer care; as others have noted, outcome measures, such as mortality and disease recurrence, do not completely describe all the various biopsychosocial consequences of a cancer diagnosis. ${ }^{3}$ To this end, several groups have developed health-related quality of life 
(HRQOL) instruments to understand the effects of disease and treatment on different domains of health in a standardized fashion. Generic instruments, such as the SF-36, permit comparison of patient HRQOL across different disease processes. ${ }^{4}$ Other instruments recognize the unique quality of life consequences of cancer diagnoses in general. ${ }^{5,6}$ More specific yet, instruments such as the FACT-M used in the current study are tailored to specific cancer entities so that the questions are as relevant as possible to that particular disease and its treatment sequelae. ${ }^{7}$

A number of studies have examined HRQOL in the context of melanoma using a mix of generic, cancerspecific, and melanoma-specific instruments. ${ }^{8,9}$ Several of these studies examine the change in HRQOL with specific chemotherapeutic agents; others compare the HRQOL of patients with advanced melanoma to other disease processes. $^{10,11}$ These studies equip physicians with the information to accurately counsel patients with new melanoma diagnoses or who are embarking on new treatment regimens regarding the nature of a typical patient experience. The Banting study is unique in that it examines HRQOL consequences for a particular surgical intervention from which the patient did not derive benefit on traditional, quantitative oncologic outcome measures. Direct investigation of the patient perspective is important because of the potential mismatch between how physicians and patients assess and value different types of information when making medical decisions regarding melanoma treatment. $^{12}$ Had the researchers found that these patients expressed significant regret, surgeons would have to alter the manner in which they educated and advised future patients weighing the risk and benefit of SLNB.

Continued work on patient-reported outcomes in melanoma will complement research on the survival benefits of medical and surgical treatment. Not only will this information enable physicians to help patients accurately anticipate their disease and treatment experience, understanding the factors that contribute to impaired or enhanced HRQOL allows for identification of potential targets to improve the quality of a patient's life, not just its length. With regard to SLNB, future work could include qualitative study of the patient perspective in order to generate hypotheses regarding the processes by which patients gain value or make meaning of their operation and its results. Ultimately, it our responsibility to invite patients to speak and, when they do, to listen.

\section{REFERENCES}

1. Banting S, Milne D, Thorpe T, et al. Negative sentinel lymph node biopsy in patients with melanoma: the patient's perspective. Ann Surg Oncol. 2019;26(7):2263-7.

2. Crossing the Quality Chasm: A New Health System for the 21st Century. Summary. http://www.nationalacademies.org/hmd/ / media/Files/Report\%20Files/2001/Crossing-the-Quality-Chasm/ Quality\%20Chasm\%202001\%20\%20report\%20brief.pdf.

3. Oliver A, Greenberg CC. Measuring outcomes in oncology treatment: the importance of patient-centered outcomes. Surg Clin N Am. 2009;89(1):17-25.

4. McHorney CA, Ware JE Jr, Lu JF, Sherbourne CD. The MOS 36-item short-form health survey (SF-36): III. Tests of data quality, scaling assumptions, and reliability across diverse patient groups. Med Care. 1994;32(1):40-66.

5. Aaronson NK, Ahmedzai S, Bergman B, et al. The European organization for research and treatment of cancer QLQ-C30: a quality-of-life instrument for use in international clinical trials in oncology. J Natl Cancer Inst. 1993;85(5):365-76.

6. Cella DF, Tulsky DS, Gray G, et al. The functional assessment of cancer therapy scale: development and validation of the general measure. J Clin Oncol. 1993;11(3):570-9.

7. Cormier JN, Ross MI, Gershenwald JE, et al. Prospective assessment of the reliability, validity, and sensitivity to change of the functional assessment of cancer therapy-melanoma questionnaire. Cancer. 2008;112(10):2249-57.

8. Cornish D, Holterhues C, van de Poll-Franse LV, Coebergh JW, Nijsten T. A systematic review of health-related quality of life in cutaneous melanoma. Ann Oncol. 2009;20(Suppl 6):vi51-8.

9. Cormier JN, Cromwell KD, Ross MI. Health-related quality of life in patients with melanoma: overview of instruments and outcomes. Dermatol Clin. 2012;30(2):245-54.

10. Cohen L, Parker PA, Sterner J, De Moor C. Quality of life in patients with malignant melanoma participating in a phase I trial of an autologous tumour-derived vaccine. Melanoma Res. 2002;12(5):505-11.

11. Long GV, Atkinson V, Ascierto PA, et al. Effect of nivolumab on health-related quality of life in patients with treatment-naïve advanced melanoma: results from the phase III CheckMate 066 study. Ann Oncol. 2016;27(10):1940-6.

12. Wong A, Billett A, Milne D. Balancing the hype with reality: what do patients with advanced melanoma consider when making the decision to have immunotherapy? Oncologist. 2019. https://d oi.org/10.1634/theoncologist.2018-0820.

Publisher's Note Springer Nature remains neutral with regard to jurisdictional claims in published maps and institutional affiliations. 